\title{
Equidad, pertinencia y relevancia educativa mediante arte, solar y lengua maya
}

\section{Equity, pertinence and relevance on education through art, the Mayan solar and Mayan language}

\section{Rosa Guadalupe Mendoza Zuany*}

El artículo analiza cómo la pertinencia, la relevancia y la equidad pueden construirse en un proyecto colaborativo, cuyo centro es el arte, el solar y la lengua maya, para transformar la educación primaria desde el ámbito comunitario; se implementó en dos comunidades en el estado de Yucatán, en articulación con sus escuelas primarias. Mediante entrevistas con los coordinadores del proyecto, sus talleristas, los docentes de las escuelas y las madres y los padres de familia, así como dibujos-entrevistas con los estudiantes y observación no participante, se obtuvieron los datos cualitativos que son analizados a la luz de las categorías mencionadas. El proyecto ha detonado procesos educativos pertinentes a partir de aprendizajes situados sumados al fortalecimiento de la lengua maya, la reconexión de los niños y las niñas con la tierra, así como mayor vinculación entre las escuelas y las comunidades; también ha propiciado una educación más relevante al contribuir positivamente al logro de los aprendizajes escolares y a una mayor equidad educativa, que ha generado nuevas expectativas sobre la educación de los niños y las niñas, así como aprendizajes in-esperados en el imaginario de las comunidades por asumirse como contextos vulnerados.

The article analyzes how pertinence, relevance and equity can be built in a collaborative project that focuses on art, the Mayan solar and the Mayan language, to transform primary education from the community level, and implemented in two communities in the state of Yucatan in articulation with its primary schools. Through interviews with the project coordinators, their workshops facilitators, school teachers, and mothers and fathers, as well as drawings-interviews with students and non-participant observation, the qualitative data was constructed and analyzed at light of the initially mentioned categories. The project has triggered pertinent educational processes based on situated learning, together with the strengthening of the Mayan language, the reconnection of boys and girls with the land, as well as greater links between schools and communities; it has fostered more relevant education by contributing positively to the achievement of school learning and has fostered greater educational equity through the emphasis on triggering new expectations about the education of boys and girls, as well as unexpected learnings in the communities for assuming themselves as vulnerable contexts.

Recibido: 4 de febrero de 2020 | Aceptado para su publicación: 30 de septiembre de 2020| Fecha de publicación: 26 de octubre de 2020

Recuperado de: https://sinectica.iteso.mx/index.php/SINECTICA/article/view/1070

doi: 10.31391/S2007-7033(2020)0055-010

\footnotetext{
* Doctora en Política por la University of York, Reino Unido. Investigadora del Instituto de Investigaciones en Educación de la Universidad Veracruzana. Miembro del Sistema Nacional de Investigadores. Líneas de investigación: educación indígena, educación intercultural, articulación de la educación intercultural y ambiental y evaluación de políticas educativas. Correo electrónico: lupitamendoza.zuany@gmail.com/https://orcid.org/0000-0002-5503-4158
}

Palabras clave:

pertinencia, relevancia, equidad, arte, trabajo con la tierra

\section{Keywords:}

pertinence, relevance, equity, art, work with the land 


\section{INTRODUCCIÓN}

$\checkmark \mathrm{l}$ artículo analiza la pertinencia, relevancia y equidad como criterios de cali-

E dad del proyecto "Arte, medio ambiente, conocimientos universales y locales claves para transformar la educación desde la comunidad", desarrollado por una asociación civil que trabaja en el ámbito educativo. Este proyecto se lleva a cabo desde 2014 en dos comunidades mayas del estado de Yucatán: Cholul y Cantamayec, municipio de Cantamayec, en articulación con las dos escuelas primarias de las localidades; la intención es poner en el centro el arte, el solar y la lengua maya para transformar la educación desde el ámbito comunitario. Este artículo deriva de una investigación realizada en 2019 que valoró cualitativamente los impactos que ha tenido el proyecto en las comunidades, las escuelas y los actores participantes; para ello, se efectuó un ejercicio de autoevaluación y un análisis que utiliza criterios de pertinencia, relevancia y equidad, que constituyen criterios de calidad (Schmelkes, 2018). Aquí solo nos enfocamos en este último análisis con base en los tres criterios de calidad mencionados.

El principal objetivo del proyecto es "lograr un proceso educativo que parta del potencial creativo de los niños y niñas para fortalecer holísticamente sus habilidades y valores a partir de su participación en espacios formativos de arte y medio ambiente" (Mejen t'aano'ob, Pequeñas Voces, AC, s.f.). Consiste en la oferta de talleres extraescolares artísticos, de lengua maya y de trabajo en los solares de las escuelas.

Los solares mayas son espacios alrededor de las viviendas destinados a actividades de subsistencia, como la producción de alimentos y la cría de animales domésticos (Cabrera, 2014). Quienes diseñaron y pusieron en marcha el proyecto buscan originar cambios positivos en la autopercepción de las potencialidades de cada individuo a través de una educación humana e integral; reconocen el valor de la cultura e identidad maya de la población con la que se trabaja, así como las necesidades y potencialidades de personas que viven en condiciones de pobreza, marginación y rezago educativo (coordinadora del proyecto).

Este artículo se concentra en el análisis de la pertinencia, relevancia y equidad, tres criterios de calidad de la educación, que en este caso son revisados en un proyecto educativo que ocurre en paralelo a los procesos educativos de las escuelas, pero paulatinamente en integración. Estos criterios han sido secundarios frente a una visión de calidad educativa hegemónica en la que prima la eficacia y eficiencia en el logro de aprendizajes relacionados con la lectura, la escritura y las matemáticas evaluados con pruebas estandarizadas. Nuestro análisis pretende visibilizar cómo dichos criterios pueden construirse en proyectos colaborativos entre iniciativas extraescolares, escuelas y comunidades. Para ello, primero abundamos en la caracterización del proyecto y el contexto; luego, presentamos los referentes teórico-metodológicos que guían el análisis, y después los resultados de cada criterio de análisis; finalmente, cerramos con la conclusión.

\section{EL PROYECTO Y EL CONTEXTO}

El proyecto desarrollado desde 2014 por una asociación civil en colaboración con las escuelas primarias tiene un diseño flexible y adaptable a las respuestas de niños, niñas, docentes y familias, y se basa en líneas base o diagnósticos de las comunidades y 
la educación que reciben los niños y las niñas que han sido realizados para diseñarlo y retroalimentarlo. Los diagnósticos de los directores de las escuelas sobre las comunidades son también un insumo valioso. Su implementación consiste en talleres de literatura, música (sensibilización, teoría musical e instrumentos), artes visuales, teatro en el aula, solar educativo, lengua maya y deportes dirigidos a niños y niñas de escuelas primarias. Los talleres se impartían inicialmente en las tardes en las dos escuelas, y poco a poco algunos se empezaron a ofrecer también en el horario escolar para intentar una articulación más efectiva entre el proyecto y las escuelas.

Los principales actores son: los tres coordinadores del proyecto que forman parte de la asociación civil (uno de ellos es director de una de las escuelas); los directores y maestros de las dos escuelas; las personas que ofrecen los talleres en las escuelas; las promotoras del solar y de teatro, que son jóvenes y madres de familia que apoyan los respectivos talleres; los estudiantes de primaria; jóvenes integrantes de los talleres de música, así como las madres de familia de los niños y las niñas, de las cuales algunas participan en el taller de lengua maya para alfabetizarse.

Los talleristas son profesionales del arte y de agroecología contratados por el proyecto, y la mayoría de ellos son originarios de la región; varios hablan la lengua maya y, a través de ella, se comunican con los estudiantes. Cuatro maestros de las escuelas se han incorporado al proyecto como talleristas: sin embargo, la mayoría de los docentes de las escuelas permanecen como espectadores del proyecto.

El proyecto tiene lugar en Cholul y Cantamayec, comunidades que se ubican en el municipio de Cantamayec, Yucatán, cuyo grado de marginación municipal es muy alto, con el $45.24 \%$ de la población que vive en pobreza extrema, según datos de 2010 (Sedesol, 2013). En 2015, contaba con 2,519 habitantes en total, y el 95.67\% de ellos eran considerados indígenas. El $85.20 \%$ de la población de tres años y más habla alguna lengua indígena y el $11.26 \%$ de esta población no habla español. En ese mismo año, el $72.1 \%$ de la población de quince años y más había cursado educación básica y el 18.3\% no contaba con escolaridad (INEGI, 2016).

Las escuelas primarias de las dos localidades son regulares, es decir, no son escuelas indígenas donde se trabaje con un enfoque intercultural bilingüe, a pesar de estar localizadas en comunidades con población hablante de maya (una población con mayor vitalidad de la lengua que otra). Una es multigrado y otra de organización completa. Si bien la infraestructura y el equipamiento de las escuelas es precario, la rotación de maestros en la primaria multigrado es alta y la atención a niños y niñas mayas no es intercultural bilingüe, en ambas primarias la comunidad escolar ha acogido el proyecto y reconoce sus aportes a una formación integral

El contexto comunitario y regional donde se desarrolla este proyecto se describe a partir de las voces de los principales actores adultos involucrados en el proyecto recogidas en entrevistas individuales y grupales, así como en la observación en trabajo de campo. Los jóvenes mencionan que en la comunidad se pueden apreciar vicios como el alcoholismo, la drogadicción o la vagancia, que no aportan beneficios económicos o emocionales (entrevista grupal con jóvenes). Señalan, asimismo, que esto se debe a que no existen en la comunidad una variedad de actividades en las cuales los jóvenes puedan ocuparse. Refieren que "no hay recurso, no hay oportunidades" (entrevista grupal con jóvenes) para quienes desean sembrar o emprender alguna otra labor. 
Por su parte, las madres de familia entrevistadas en grupo (entrevista grupal con madres 1) expresan que sus hijos solían andar en las calles de las comunidades sin alguna ocupación, veían televisión en las tardes, eran penosos y no se comunicaban con facilidad. En Cantamayec, en particular, ellas reconocen no enseñarles a hablar la lengua maya a sus hijos. Mientras que en Cholul el 100\% de los niños hablan la lengua (director 1), en Cantamayec menos niños la hablan (o lo niegan) (entrevista grupal con madres 1). Las madres también reconocen en la entrevista grupal que los conocimientos sobre actividades agrícolas o del solar ya no son compartidos por toda la comunidad y, por ende, tampoco por los niños.

Por su parte, los maestros de las primarias describen un contexto de marginación, aislamiento y pobreza que se traduce en falta de oportunidades. La estructura familiar se ha visto afectada por la migración de los padres a zonas urbanas: "Las familias se separan porque los papás tienen que viajar a trabajar, niños que se quedan con los abuelitos, se van a Mérida, Cancún, Holbox" (maestro 5). El abandono de actividades agrícolas y ganaderas ha mermado la transmisión de conocimientos locales sobre el trabajo con la tierra, a juicio de los coordinadores del proyecto (maestro 8).

Un docente establece una relación directa entre desempeño académico y marginación, concretamente expresada en desnutrición: "La gente aquí trabaja para sobrevivir, para subsistir; para nosotros es muy palpable ver cómo llegan los niños a la escuela, hay niños que vienen a comer, ya ve que estamos en un programa de tiempo completo con alimentación" (maestro 9). Identifican rezago educativo y pocas expectativas de seguir estudiando después de la secundaria, e incluso de la primaria: "Yo he estado aquí siete años, ninguno de mis alumnos ha terminado todavía una carrera, la mayoría fue desertando" (maestro 6). Lo anterior se agudiza en el caso de las niñas, pues se desenvuelven de acuerdo con lo que describen varias maestras como un contexto machista que limita sus aspiraciones a permanecer en casa e, incluso, genera violencia doméstica.

Por otro lado, en las escuelas, la mayoría de los maestros se enfocan en los aprendizajes del currículo referidos a la lectura, escritura y matemáticas, y en evaluarlos, con clases de educación artística precarias, sin planeación ni consideradas importantes: "A nosotros nos piden 'mide lectura, mide lectura, mide matemáticas, mide ciencias naturales, mide historia', si el niño no sabe historia, repruébalo y listo y los docentes solo vemos contenido porque se me está acabando mi tiempo" (maestro 1). Sobre el currículo de educación artística de la Secretaría de Educación Pública (SEP), dice: “QQué hace la maestra de artísticas? [...] es que ellos no tienen un programa y cada maestra ve lo que Dios le da a entender" (maestro 1).

El panorama descrito por los propios actores comunitarios y educativos fundamenta la propuesta y la implementación de un proyecto que busca reposicionar el solar y la lengua maya en los procesos educativos, así como colocar el arte como un elemento central en la vida de los niños, las niñas y las comunidades en general. Coordinadores y talleristas hablan de una formación integral que dote a los niños y las niñas de conocimientos para transferirlos a su vida.

\section{REFERENTES TEÓRICO-METODOLÓGICOS}

El análisis del proyecto se basó en tres criterios de calidad: pertinencia, relevancia y equidad (Schmelkes, 2018), y se fundamenta en la propia naturaleza abierta y 
flexible del proyecto para adaptar sus objetivos y estrategias a las condiciones de las comunidades y las escuelas, así como a las necesidades, ritmos y aprendizajes de todos los involucrados. La intención es explorar lo que cualitativamente se ha logrado al tener como guía estos criterios y cómo lo han hecho.

El proyecto, a través de sus talleres, busca detonar aprendizajes y capacidades referidos al trabajo con la tierra y con la expresión artística, todo a través del uso de la lengua maya para su fortalecimiento. Para analizarlos, el concepto de pertinencia permite observar el proyecto e identificar si atiende y cómo atiende necesidades y demandas de los actores que emergen de lo local y cotidiano.

La pertinencia se entiende como un atributo de un proceso educativo que es intersubjetivo, contextual y situado históricamente en el que se consideran las demandas y expectativas de diversos actores educativos (Cabrera, 2019), desde un nivel que inicia en lo individual, pues parte de las características, particularidades y necesidades de los sujetos y abarca también a la sociedad (Schmelkes, 2018). En general, se alude a la pertinencia cultural y lingüística como un medio para combatir la asimetría escolar que se expresa en aprendizajes que no les sirven para su vida, sobre todo si se trata de una vida comunitaria (Schmelkes, 2003). La asimetría escolar expresada en indicadores de logro académico tiene referencia directa con la pertinencia:

La asimetría escolar se combate ofreciendo una educación de calidad a los indígenas en todos los niveles educativos, desde el preescolar hasta la universidad. Calidad significa ofrecer una educación cultural y lingüísticamente pertinente. Desde el paradigma de la diversidad, la calidad debe alcanzarse por los caminos más adecuados, que difieren según los grupos culturales y contextos poblacionales (Schmelkes, 2003, p. 7).

La calidad se concreta a través de aprendizajes situados que ocurren porque "el conocimiento es situado, es parte y producto de la actividad, el contexto y la cultura en que se desarrolla y utiliza" (Díaz-Barriga, 2003), de procesos educativos vinculados a la comunidad (Jiménez, 2009) y de la apertura a definir y propiciar aprendizajes in-esperados, es decir, que no han sido definidos desde el currículo oficial como "esperados" (Mendoza, 2020) que emergen del contexto social y cultural.

Por su parte, la relevancia alude a lo que es importante aprender cuando se ubica a los alumnos más allá de lo local y que incluso es utilitario. Concierne a las "necesidades" de la sociedad en múltiples escenarios, como la "productividad económica", "la democracia política", el "respeto a los derechos humanos", "el cuidado del ambiente y la preservación y enriquecimiento de la diversidad cultural" (Schmelkes, 2018), entre otros temas socialmente compartidos. La relevancia se asocia al logro de aprendizajes clave en los planes de estudio, definidos con base en los fines de la educación a nivel nacional y global.

La articulación de pertinencia y relevancia plantea la búsqueda de "equilibrios a alcanzar: entre lo mundial y lo local, o entre lo universal y lo singular", entre "convertirse en ciudadano del mundo y participar activamente en la comunidad de origen" o "entre las necesidades del mercado del trabajo y las del desarrollo personal" (Unesco, 2007), de manera que los niños y las niñas logren un desarrollo personal y comprendan y se conecten con su comunidad, sin ignorar los fenómenos fuera de esta, puesto que inciden en las dinámicas locales.

La equidad como criterio de calidad se refiere a que "las oportunidades y resultados educativos no dependen de atributos adscriptivos como el lugar de nacimiento, 
el género, la condición étnica, el nivel de ingreso del hogar o el nivel educativo de las personas encargadas del cuidado y la socialización del niño-joven" (Blanco, 2019, p. 30). Esto se alcanza con acciones públicas y educativas que apoyen a quienes lo requieren para ejercer su derecho a la educación de calidad.

Este criterio es importante en contextos vulnerados donde los "mínimos" de calidad son considerados "techos". En ese sentido, se concretan mediante acciones educativas que expandan los mínimos que se expresan al hacer mayor hincapié en lectura, escritura y matemáticas hacia ámbitos considerados como lujos para niños y niñas en contextos vulnerados, como puede ser el artístico y el del fortalecimiento de su lengua materna no solo de forma oral, sino también escrita.

Partiendo del derecho a la educación, caracterizado en las cuatro A de Tomasevsky (2004), los tres criterios mencionados fundamentan un análisis que se enfoca en la adaptabilidad referida a la relevancia de la educación para poblaciones diversas y en la aceptabilidad relacionada con la utilidad de lo aprendido en un contexto escolar de respeto y equidad (Schmelkes, 2018). La equidad tiende a asociarse a la accesibilidad y disponibilidad; sin embargo, esta depende también de los aprendizajes pertinentes y relevantes, sin obstáculos u omisiones para su logro por su condición social y étnica, más aún, con apoyos para obtenerlos.

El análisis del proyecto con base en las categorías conceptuales abordadas implicó fundamentarse en un posicionamiento epistemológico constructivista y crítico (Guba y Lincoln, 2012), que se expresa en la interpretación de datos cualitativos construidos intersubjetivamente $\mathrm{y}$, a la vez, en un análisis que se orienta a incidir en el proyecto para retroalimentarlo en concordancia con sus participantes y con la intención compartida con ellos de transformar la educación de las comunidades.

La construcción de datos se llevó a cabo por medio de entrevistas, observaciones y análisis de documentos del proyecto. Las entrevistas las entendimos como espacios de construcción de conocimiento entre los entrevistados y el entrevistador en contextos y situaciones concretas (Kvale, 1998). Realizamos 30 entrevistas semiestructuradas a los actores adultos (coordinadores del proyecto, directores y maestros de escuelas, y talleristas) con un guion de temas que permitió la emergencia de preguntas a partir de respuestas; así, logramos enfocar las preguntas en el abordaje de las categorías de análisis de manera progresiva (Flick, 2004).

También, hicimos entrevistas estructuradas grupales, una con jóvenes de ambas comunidades y otra con madres de familia, en las que utilizamos guiones con preguntas concretas para todos los participantes. Con los 30 niños y niñas participantes en los talleres, recurrimos al dibujo-entrevista (Lomax, 2012), que nos ayudó a construir datos sobre escenarios de su propio futuro. Efectuamos la observación no participante en una sesión de cada uno de los talleres ofrecidos en una estancia de una semana en las comunidades y revisamos documentos del proyecto, sobre todo su historia y propuestas fundacionales.

El análisis de los datos construidos implicó respetar los sentidos plasmados en las voces de los participantes y la complejidad de representarlas por escrito. Procuramos un alejamiento de la idea de validación de datos cualitativos a través de la triangulación (Denzin, 1970) y de una cristalización de datos, que "permite mostrar que no existe una verdad singular, [...] la cristalización nos proporciona una comprensión de 
los temas, parcial, dependiente y compleja" (Richardson, 1997, p. 92) y que no existe un punto fijo verdadero para interpretar el mundo desde el cual triangular lo dicho y lo observado (Richardson y Adams, 2016).

Llevar a cabo lo anterior implicó que nuestra postura sustentada en educación intercultural e indígena fuera visible y que nuestras interpretaciones no se expresaran desde una posición de autoridad y verdad. Del mismo modo, significó introducir las voces de los participantes del proyecto para mostrar su complejidad y la diversidad de perspectivas presentes. Para lograrlo, con el uso de distintos métodos de construcción de datos (entrevistas, dibujos-entrevistas y observación no participante), contrastamos lo dicho y lo observado para dilucidar la complejidad incluso por parte de un mismo sujeto y entre ellos en el marco de un proyecto común. También, formulamos las mismas preguntas a diferentes actores para cotejarlas e identificar tensiones y acuerdos a fin de reconocer la diversidad de los actores involucrados.

Para examinar los datos, cada criterio de calidad fue considerado una categoría, y de cada una derivaron subcategorías que se concretaron en nociones y prácticas enunciadas u observadas durante el trabajo de campo. Esto no implicó buscar generalizar a través de hallazgos que presentaran una perspectiva única y verdadera de las formas de construir pertinencia, relevancia y equidad, sino mostrar múltiples maneras de entenderlas y procurar alcanzarlas. Con ello, desde el análisis, pretendimos comprender el modo específico en que se construye la calidad con base en las intenciones, acciones y logros del proyecto.

\section{ANÁLISIS A TRAVÉS DE LOS CRITERIOS DE CALIDAD}

\section{Pertinencia}

El proyecto posiciona dos elementos que dotan de pertinencia y que han sido relegados del proceso educativo en las escuelas con las que colabora: la lengua maya para su aprendizaje en el caso de niños y niñas que no la usan y para la alfabetización de quienes sí la utilizan a diario, así como el trabajo con la tierra en el solar educativo. Estos elementos cotidianos en un contexto rural se han abordado en talleres que buscan detonar diversos aprendizajes situados, no solo en los niños y las niñas, sino en sus madres, que también se alfabetizan y participan como promotoras de solares en la comunidad. Se trata de visibilizar ante ellos la importancia de prácticas y conocimientos que han sustentado la vida de sus comunidades de manera histórica a través de su propia lengua y su vínculo con la tierra.

Como señala Cabrera (2019), la pertinencia se construye a partir de demandas y expectativas, que en el marco del proyecto han sido recogidas por medio de varios actores comunitarios, que incluyen a niños y niñas, madres y docentes, a los que reconocemos como sujetos con voz y expectativas; por ejemplo, hemos trabajado en la adecuación y la flexibilidad del proyecto ante un contexto y actores que "comunican" con sus propias voces lo que necesitan y demandan; esto ha abierto la posibilidad de que los alumnos del proyecto expresen lo que quieren y tengan la oportunidad de elegir. 
Por su parte, la identificación de necesidades ha emergido de diagnósticos aplicados para valorar los impactos del proyecto y determinar estrategias y talleres específicos, así como de la observación que realizan coordinadores y talleristas del proyecto y que interpretan con sus propias visiones de la pertinencia y la importancia que atribuyen a lo local y de los problemas que el proyecto puede resolver, o no; de ahí la relevancia de que sean talleristas originarios de la región. Los comportamientos, sucesos y problemáticas que observan en los niños, en la escuela y la comunidad, por ejemplo, la discriminación de género, la necesidad de producir alimentos sanos o de hablar de temas sensibles, como las divisiones políticas en la comunidad, son insumos valiosos para lograr lo pertinente y relevante.

La pertinencia educativa, en específico la lingüística, no solo es un criterio de calidad de la educación; constituye un derecho. Dado que las escuelas con las que colabora el proyecto no son interculturales bilingües, a pesar de operar en comunidades eminentemente indígenas, destaca que el proyecto abone a que los niños y las niñas tengan espacios formativos en y sobre la lengua maya. Las escuelas no están obligadas a una atención educativa en lengua maya y con algunas excepciones es usada por maestros hablantes de maya que son sensibles a la trascendencia de que los niños y las niñas reciban una educación en su lengua como es su derecho.

En este sentido, la pertinencia lingüística se construye mediante tres rutas. La primera es con el taller de maya a los niños y las niñas. En Cholul, el taller ha contribuido al surgimiento de un proceso de alfabetización de quienes portan el maya como primera lengua, que constituyen, a juicio del director, un $100 \%$ del alumnado de la escuela. En Cantamayec, donde muchos niños ya no la aprendieron como lengua materna, ofrece una oportunidad para aprenderla y valorarla. En ese contexto ha sido relevante para los niños que sí hablan maya y que, al mismo tiempo, no han sido los más aventajados en la escuela, su visibilización y valoración por sus conocimientos de lengua entre sus demás compañeros. El taller de maya ha permitido hacer notorio diferentes conocimientos y capacidades del alumnado. Sobre los que hablan maya, el maestro asegura:

Cuando metieron maya, esos niños son muy mayeros, ellos son como que los inteligentes del salón, los que más participan porque todo lo que pregunta el maestro, ellos lo saben. Ellos hasta se burlan de sus compañeros, porque hay compañeros que no saben ni una palabra, entonces cuando el maestro habla maya, ellos son hasta como intérpretes y entonces ellos ya se siente en el salón importantes porque ya el maestro ya los vio y ellos son como sus ayudantes, los agarra de ayudantes, y ellos se sienten como importantes dentro de la clase porque ellos sí saben maya. Ellos cambian los papeles: los que los ayudan siempre en las otras materias, ahorita ellos requieren su ayuda de ellos (maestro 8).

La segunda ruta de construcción de pertinencia lingüística han sido los talleres cuyos participantes pueden comunicarse en maya. Para los talleristas, tanto mayahablantes como no, es importante la comunicación con los niños y las niñas en maya: "Yo soy maya hablante, entonces, yo llego a darles clases de música en lengua maya y yo siento que se empapan de música, porque puedo llegar y hablarles directo a ellos y deja de haber esa barrera de comunicación" (tallerista 2). La formación del equipo idóneo ha implicado considerar y valorar en especial a talleristas de la región, varios de ellos mayahablantes, originarios de comunidades vecinas y que comparten conocimientos y prácticas culturales con los estudiantes, como lo señala el coordinador: "Lo que no funcionó fue traer gente de fuera" (coordinador 3). 
Aparejado al uso del maya en los talleres, los talleristas destacan el abordaje de saberes y prácticas locales y contextualizadas en su propia lengua para un aprendizaje situado (Díaz-Barriga, 2003), como se hace en el solar:

Con los niños tratamos de rescatar saberes campesinos, que igual que el maya, se viene perdiendo, porque ya hay pocos hombres que trabajan el campo. Nosotros tratamos, con el solar, de rescatar la importancia de sembrar, el trabajo de campo, la alimentación y de que siga permaneciendo ese trabajo. También se logra tener diferentes trabajos como recetario, calendario de producción, fichas técnicas y materiales ilustrativos que servirán para compartir con los niños/niñas que se llevarán para la casa o quedarse en la biblioteca de la escuela para su consulta. Nosotros hacemos equipo en maya, tratamos de hacer que ellos aprendan, que igual identifiquen nombres de cultivos en maya (tallerista 5).

El solar es un espacio perfecto, desde el punto de vista de un tallerista, para el contacto y aprendizaje de miembros de la comunidad, de forma situada y valorando los saberes locales y a quienes no se visualizan como sabedores desde la escuela:

No solo permanecemos en el espacio del solar como tal, los niños son los que proponen; tenemos un plan, dentro de ese plan cae lo que proponen ellos. De repente se nos ocurrió, "vamos a visitar el solar de cada uno", entonces, me doy cuenta que les gusta mucho salir. Hay un montón de ejemplos donde te topas con diferentes saberes, con gente que trabaja con plantas medicinales. Eso del puente intergeneracional que se está perdiendo, por ejemplo, de la importancia de valorar el saber del abuelito (tallerista 4).

Las estrategias para valorar los conocimientos locales y a sus portadores han sido diferenciadas, pero una que ha sobresalido es la visita a los solares de las casas de los alumnos. Esta experiencia ha sido muy enriquecedora, descubridora de saberes en contacto con la comunidad, y desmontadora de prejuicios, como el que señala que en la escuela es donde se aprende:

En Cantamayec se buscó otra estrategia, ahí se ha buscado desde el solar salir a caminar con los niños, [...] te empiezas a encontrar con mujeres o con familias que tienen un potencial increíble para alimentar el solar. Entonces sí es un proyecto que sale a vincularse con la comunidad. Aunque puede haber menosprecio de los maestros hacia la comunidad, el proyecto sí impulsa el que dejemos ese menosprecio, ese racismo atrás, y creamos que las comunidades tienen un montón de potencial (coordinador 2).

La pertinencia se logra mediante un proceso intersubjetivo (Cabrera, 2019) en el que no solo los talleristas aprecian los aprendizajes situados y útiles en la comunidad, sino también en el que los niños y las niñas consideran lo aprendido. El taller de solar educativo es un espacio de vinculación escuela-comunidad (Jiménez, 2009) especialmente valorado por los estudiantes. Un niño menciona que le gusta sembrar, una actividad que hacen sus padres; él se siente "bien porque ya también sé sembrar"; en su casa, ahora siembra "remolacha, tomate, cilantro, rábano, zanahoria" (dibujo-entrevista 1). Una niña indica que "ya puedo sembrar bien", al compararse con el inicio del taller cuando no sabía "nada" (dibujo-entrevista 12).

Las madres de familia, muchas de ellas muy jóvenes, expresan que les gusta que sus hijos tengan taller de solar, pues antes no sabían sobre el cultivo de la tierra; ahora los niños han comenzado a sembrar también en sus casas y les platican y enseñan a ellas (entrevista grupal con madres 1). Los solares han permitido que se valoren y reposicionen saberes que las comunidades consideraban poco valiosos y que no se animaban a compartir en la escuela. La visita a solares y el reconocimiento de los saberes de miembros de la comunidad generan una dinámica 
distinta y contribuyen a la autoestima de los niños y las niñas que muestran lo que su familia sabe hacer cuando visitan sus casas.

Los aprendizajes in-esperados (Mendoza, 2020) en las escuelas, aunque se ubiquen en zonas rurales y campesinas, y que sí se detonan en los talleres, son observados con sorpresa por los docentes. Una de ellas habla de lo que advierte en sus alumnos pequeños que trabajan en el solar: "El huerto los tiene fascinados. Me tocó ver que cosechaban. Me llamó mucho la atención que ellos mismos cultivan" (maestro 2). El carácter inesperado de estos aprendizajes origina la desconexión en la práctica educativa entre las escuelas y las comunidades, pero, al mismo tiempo, estimula la reflexión sobre una práctica educativa situada que atienda y reconozca el contexto donde ocurre.

Lo anterior implica partir del valor de los conocimientos y las prácticas que poseen los habitantes de las comunidades y no de una caracterización negativa de comunidades donde es palpable su marginación y vulnerabilidad por procesos económicos, sociales y culturales que responden a fenómenos extracomunitarios. Así, un aspecto positivo del proyecto que también se resalta es la apertura y vinculación con la comunidad, que se valora como idea fuerza y también como proceso inacabado. Dicha vinculación no se refiere solo al solar, sino a actividades que han sido consideradas un "lujo" para comunidades, como el arte. En este sentido, una de las coordinadoras afirma:

Una idea fuerza es la apertura a la comunidad, sí estamos luchando y muy atentas a cualquier rasgo de menosprecio hacia la comunidad. Los profes y los talleristas, todos, me parece que han sido bastante abiertos y sensibles a que hay que salir. También podría convertirse el arte en algo bien exclusivo de los niños y encerrarse, pero el arte tiene que salir y sus exposiciones salen y sus espectáculos salen, su música sale, lo que hacen en el solar sale. Esa es otra idea que tiene que estar permeándose entre la comunidad y la escuela (coordinador 2).

De ese modo, la pertinencia se construye en el proceso educativo y se valora, intersubjetivamente, el contexto, los conocimientos y las prácticas comunitarias vigentes, y también aquellas que ya no alcanzan a los niños y las niñas en sus hogares ni tampoco en las escuelas, que califican muchos de estos aprendizajes que emergen en los talleres como in-esperados, en particular en contextos donde se tiende a resaltar ciertos aprendizajes básicos, como la lectura, la escritura y las matemáticas, por considerarlos elementales y suficientes para sobrevivir, desde una perspectiva que no considera la pertinencia como un criterio de calidad y como expresión de su derecho a la educación.

\section{Relevancia}

La relevancia es un criterio de calidad que apunta a que los alumnos logren aprendizajes que sirvan más allá del contexto local, que posibiliten que se ubiquen en un escenario extracomunitario. Si bien la relevancia y la pertinencia generarían un balance entre los aprendizajes que necesita un ciudadano en cualquier contexto y en lo comunitario y local, lo que ocurre en las escuelas es mucho más complejo. Los docentes tienden a concentrarse en los aprendizajes de lectura, escritura y matemáticas, debido a que las mediciones ubican a sus escuelas en rezago educativo y bajo logro. 
Aun cuando es importante que obtengan estos aprendizajes sujetos a medición, concentrarse en ellos y dejar de lado otros, por ejemplo, los artísticos, vulnera su derecho a la educación de calidad, integral, independientemente de su origen étnico o de su condición socioeconómica.

Buscando un equilibrio entre pertinencia y relevancia, el proyecto se propuso articular y vincular la formación que se impulsa en los talleres con los contenidos curriculares que dotan de relevancia a la educación. Este ha sido un reto, ya que, para lograr el apoyo de los docentes hacia el proyecto, fue necesario mostrarles que la participación de los niños y las niñas en los talleres les permite obtener aprendizajes no abordados ni esperados en la escuela, como tocar instrumentos musicales; actuar en obras de teatro; leer y escribir creativamente; dibujar con el uso de varias técnicas; escribir y leer en lengua maya; sembrar y cosechar o cuidar abejas meliponas. También, repercute de manera positiva en su desempeño escolar.

Una estrategia implementada por algunos talleristas para vincularse al logro de los objetivos de aprendizaje escolares es la comunicación y coordinación con los docentes para llevar a cabo actividades que se complementen. Uno de ellos comenta:

Yo le pregunto a los maestros qué están viendo, "que vamos a ver biografías", dicen los maestros, bueno entonces vamos a ver esta serie que tengo de biografías, un ejercicio que tenga que ver con biografías maravillosas; yo siempre les pregunto a los maestros de lo que están viendo para poderlo empatar, pero sobre todo en contenidos (tallerista 1).

Otro tallerista ejemplifica cómo aborda en maya los temas que están tratando los maestros de aula y otros talleristas para atender necesidades y adaptar su planeación por el carácter flexible del proyecto:

Me acoplo a lo que los maestros a veces piden en el plan de estudio. Por ejemplo, en Cantamayec, a veces los maestros dicen "vamos a ver partes del cuerpo, ¿usted lo puede igual verlo en maya?". Tenemos esa libertad de plantear temas con los niños y a veces cuando hay necesidad de un tema en específico, nosotros hacemos todo ese cambio, o sea no planeamos, por ejemplo, un mes y es obligatorio verlo. En el caso de nosotros llevamos cuentos, cualquier información en lengua maya con los niños para complementar el trabajo del maestro, pero aparte de ello, vemos temas de la comunidad. En Cholul igual trabajo con el área de solar y con la parte de teatro (tallerista 3).

Hoy, los docentes reconocen la contribución de los talleres al logro de los aprendizajes y cuando se trata de compartir al frente del salón y de escribir por sí mismos. Al respecto, un docente opina:

Ellos logran desarrollar mejor su manera de escribir, de plasmar sus textos propios, y entonces ahí van desarrollando la comprensión lectora, entonces sí les sirve también para expresarse. Los talleristas les piden que pasen al frente y hagan alguna actividad que les genera confianza; a partir de esa confianza, cuando tú les dices "pasa al pizarrón a resolver esto", también pasan (maestro 7).

Al respecto, otro docente afirma: "Les da mucho más conocimiento cuando se aborda un contenido, participan más, tienen una idea de lo que han hecho en los talleres. Tienen más interés porque ya saben más cosas. Les da más facilidad realizar las actividades" (maestro 2). La contribución al logro de los aprendizajes, también en opinión de los docentes, se relaciona con la colaboración que, en algunos casos, se ha establecido entre talleristas y maestros. La comunicación maestros-talleristas es reconocida como un aspecto que tiene efectos muy positivos, por ejemplo: "Veo que hay mucho que se 
articula, veo que sí trabajan y eso en socioemocional sí me ayuda porque trabajan en equipo. Con los que no saben leer me ayuda mucho" (maestro 4).

La contribución del proyecto a la relevancia de la educación que reciben los niños y las niñas es un mayor logro de aprendizajes y del desarrollo de habilidades y confianza en sí mismos: "Creo que los niños desarrollan muchas habilidades que son necesarias para aplicar en la escuela como la confianza, la seguridad, la creatividad, el ingenio, las clases de artes y el trabajo con la tierra" (tallerista 1). Estos resultados son cruciales para la integración paulatina de la escuela y el proyecto, y para dejar de concebirlos como ámbitos separados:

Conforme ha pasado el tiempo, los maestros de la mañana se han interesado en el proyecto y esto ha generado dos tipos de acercamiento: el arte sí dinamiza y hace más interesante el trabajo del maestro y pide ayuda, y por otro lado en Cantamayec hay testimonios de los maestros de que los niños se han vuelto más participativos, tienen más ganas de aprender, aunque es a nivel empírico [que] se han visto resultados. De una separación mañana-tarde a un acercamiento, de hecho, en las dos escuelas ya se trabaja con los talleristas por las mañanas en literatura y lectura creativa y música (coordinador 1).

La relevancia entendida como el criterio referido al logro de aprendizajes generales clave que son establecidos en el currículo nacional es prioritaria para los docentes. El proyecto ha sido determinante para mostrar a los docentes, las familias y la comunidad la importancia de una formación más allá de lo que la escuela propone como aprendizajes clave homogéneos y generales sin atención a la diversidad. El balance entre pertinencia y relevancia se expresa en el reconocimiento del valor de un proceso educativo que rebase los "mínimos" que plantean las expectativas que se tienen de niños y niñas en contextos vulnerados.

Esta vulnerabilidad debe revertirse y, a la vez, transformarse en el imaginario de los niños y las niñas mediante el acceso a aquello a lo que tienen derecho y nunca han gozado. Por ello, es crucial que la educación sea adaptable a la diversidad y aceptable en términos de lo útil de los aprendizajes generados en un marco de equidad.

\section{Equidad}

La inequidad se expresa de múltiples maneras en las comunidades y vulnera el derecho de los niños y las niñas a una educación de calidad. La marginación de las comunidades y las escuelas ha reducido las oportunidades de la niñez a recibir la atención educativa que, por derecho, le corresponde; así, los "mínimos" de otros contextos se convierten en "máximos" apenas alcanzables. Para que la equidad sea una realidad, es preciso diseñar e implementar acciones que apoyen de modo prioritario a quienes se encuentran en desventaja (Mendoza, 2019).

Además de no recibir una educación pertinente en las escuelas que potencialmente podría concretarse con un enfoque intercultural bilingüe, la relevancia mediante aprendizajes clave se reduce y delimita al generar una educación inaceptable. El acceso y la disponibilidad no deben ser los únicos criterios considerados cuando se habla de equidad; es preciso considerar si se ha adaptado al contexto y si es aceptable en términos de no limitar las oportunidades de aprender más allá de leer, escribir, sumar y restar por tratarse de contextos vulnerados. 
En este sentido, la equidad supone detonar y fomentar aprendizajes in-esperados en niños y niñas que viven en contextos de marginación, como los referidos al arte y al trabajo con la tierra. Además de apoyos que requieren recursos económicos necesariamente, es conveniente reemplazar las bajas expectativas que se tienen sobre el presente y el futuro de los niños y las niñas. Tanto talleristas como docentes coinciden en que, en el imaginario de la comunidad, no hay expectativas educativas más allá de la primaria, y se sustituyen con la migración para trabajar en centros urbanos. Esto, a su vez, ha originado cambios importantes en lo comunitario, por ejemplo, el abandono de las actividades agrícolas por parte de los hombres que migran y la pérdida de autosuficiencia alimentaria de las familias. A ello se suma que los conocimientos locales, como los relacionados con el solar, tienden a ser excluidos de las escuelas.

Ante la situación, el proyecto ha posibilitado el acceso de niños/niñas/jóvenes al logro de aprendizajes que tienen que ver con el arte y con aspectos que han sido desdeñados en su propio entorno, como el solar. Las estrategias para promover equidad en este sentido han funcionado como catalizadores para aprender como "los demás", como "los privilegiados", sin límites que se imponen -y, en ocasiones, se autoimponen las comunidades- por las condiciones de marginación (Mendoza, 2019).

La construcción de expectativas de futuro en los niños y las niñas ha sido clave en el camino hacia la equidad. Por una parte, los talleristas destacan una mayor equidad educativa al mostrar que la pobreza no determina lo que la niñez es capaz: desarrollo de habilidades y actitudes como la toma de decisiones, y la posibilidad de trabajar aspectos emocionales y de conducta mediante el arte y el solar. Mientras los maestros de aula son "tradicionales", "con el niño que llega se sienta, escucha y listo, donde el niño no tiene oportunidad de preguntar, ni de reflexionar" (maestrotallerista 1), en el proyecto la dinámica de los talleristas es diferente, "porque le estamos abriendo un mundo totalmente diferente a los niños" (maestro-tallerista 1).

Desde el punto de vista de un docente, los talleres son innovadores por ofrecerse en un contexto de marginación, donde nunca se pensó poder tener acceso a instrumentos musicales y aprender a tocarlos o a dibujar; además, para los estudiantes, las actividades que plantean los talleres son novedosas al contrastarlas con lo que los docentes de la escuela proponen: "Veo que los niños que son difíciles o que tienen problemas de conducta se integran a esas actividades. [El taller] les llama la atención; todas las actividades que buscan los maestros siempre son llamativas, vienen con una actividad nueva" (maestro 8). La innovación deriva de la novedad que el proyecto supone para la escuela y la comunidad; es una innovación respecto a lo que antes se experimentaba en ese entorno específico, a juicio de uno de los directores:

Para mí todo es innovador porque no se aplicaba nada de lo que el proyecto nos trae acá, o sea para la comunidad es nuevo. Cuando me preguntan si es innovador, sí lo es porque el niño no tenía nada en la tarde, la maquinita tal vez o en su casa con los juguetes que pudiera tener o llevarlo a la milpa a cortar leña. Se abre este proyecto tan innovador y el niño ya tiene la oportunidad de distraerse, que venga a jugar pintura o a pararse a jugar. Él anda contento, nunca lo vemos molesto o preocupado porque no sabe hacer su tarea. Al contrario, lo ven como un juego y entre ellos mismos se van aplicando (director Cantamayec).

Al abrirles la posibilidad de un mundo diferente, los niños y las niñas se imaginan un futuro propio más allá de lo convencional: "Hace un año usted le preguntaba ¿qué 
quieres ser de grande?' y no se lo imaginaban. Ahora dicen 'pintora', 'yo quiero trabajar mi huerto', 'yo quiero ver a las abejas'. Ya ha cambiado la mentalidad de los niños" (maestro-tallerista 1). Una educación sin prejuicios sobre sus límites ha sido clave:

Creo que la importancia del proyecto radica en la posibilidad de ofrecerle a los niños otro tipo de mirada, que no lo ofrece la educación pública. Creo que la educación pública está demasiado contaminada por los prejuicios de los propios maestros, que en muchas ocasiones ven en los niños como pocas posibilidades de avanzar, determinando que al estar en una comunidad pues no hay mucho qué hacer. Creo que el proyecto ofrece una mirada a través del arte, que es lo que trabajamos, en este caso de la lectura, de que los niños puedan ver otro tipo de realidad y por lo mismo tener la posibilidad de tomar otras decisiones. El arte te da la mirada de poder de repente tener otra posibilidad de crear y entonces yo creo que lo que el proyecto hace es poner un poco esa semilla en los niños (tallerista 1).

Una de las transformaciones más importantes referidas a la construcción de equidad es el cambio en las expectativas de los directores, docentes, padres y madres sobre sus hijos e hijas y de los propios niños y jóvenes sobre su futuro. Uno de los directores indica al respecto: "Las actividades realizadas por los alumnos han demostrado que pueden salir adelante, que romper aquel tabú donde la mujer no puede salir adelante, no es imposible" (director Cantamayec). En este mismo sentido, el segundo director y coordinador del proyecto menciona: "Los niños que egresan de la primaria pueden integrarse a cualquier tipo de escuela ya que poseen lo necesario para poder desarrollarse en cualquier ámbito y esto se debe a la vinculación: escuela-proyecto" (director-coordinador 3).

Las decisiones que toman y los sueños que imaginan los niños y las niñas desmontan la idea de que la pobreza condiciona, determina y limita lo que pueden lograr. Ellos mencionan sentir transformaciones personales positivas relacionadas con el desarrollo y la confianza en sus capacidades artísticas y de aprendizajes de conocimientos escolares y no escolares (como los del trabajo con la tierra). Al preguntarle a una niña cómo se sentía de saber tocar un instrumento, respondió: "Estoy feliz porque ya lo aprendí" (dibujo-entrevista 3). Otra menor que toca la flauta mencionó: "Ya me siento mejor porque al principio creí que no lo lograría, porque en la primera clase se me hacía muy difícil" (dibujo-entrevista 7).

Este cambio es valorado no solo en las actividades del taller. Uno de los niños aseguró que desde que asiste "aprendo más" en "todo", incluso en "las clases normales de la mañana”. Otra niña refirió que "ya aprendí más” (dibujo-entrevista 12) y no solo de música, su taller favorito, sino de "todo".

La transformación es observada también en las prospectivas, puesto que los niños y las niñas afirman que lo aprendido en los talleres ha incidido en sus aspiraciones a futuro. Una niña expresó que "de grande" le gustaría ser "pintora" (dibujo-entrevista 22), porque el taller de pintura le ha "ayudado a decidir". Otra destacó una aspiración similar: "Porque puedo dibujar y pintar todo" (dibujo-entrevista 23), y una más comentó: "Quiero ser pintora de grande" (dibujo-entrevista 48). Este deseo surgió de que "aquí aprendí a pintar y a mí me gustó” (dibujo-entrevista 48).

La equidad se expresa no solo en apoyos económicos que compensen o impulsen más oportunidades y mejores resultados educativos, sino en visiones de presente y futuro que reconocen el potencial de los niños y las niñas de lograr lo que se propongan a través de una educación de calidad sin importar donde vivan, su lengua 
materna o su condición socioeconómica. El proyecto dota a la equidad de una dimensión cualitativa que permite analizar este criterio con unos lentes distintos a los de la asignación de recursos y resultados favorables en la medición de aprendizajes con pruebas estandarizadas.

\section{ConCLUSIón}

En la experiencia analizada, identificamos, en primer lugar, cómo la pertinencia, relevancia y equidad se logran a partir de prácticas que se incorporan en procesos educativos. El proyecto ha establecido vínculos con la comunidad por medio del solar, que es la fuente de un tipo de saberes locales/campesinos históricamente excluidos de la escuela. El trabajo en solares es relevante para la sustentabilidad social y ambiental de las comunidades, donde muchas problemáticas se asocian a la desconexión con la tierra, al abandono de las milpas y a la inexistencia de solares como espacio de producción de alimentos y plantas para el autoconsumo en muchas de las casas de las comunidades.

En segundo lugar, encontramos que la lengua maya tiene un lugar primordial en el proyecto, ya que considera la importancia de la revitalización de la lengua maya y la cultura local. Es muy positivo que los talleristas conozcan y sean originarios de la región y contribuyan al proyecto con empatía y cercanía desde el punto de vista cultural, que recurran a su propio conocimiento del medio, de la lengua maya, y de sus experiencias personales y profesionales.

Otro logro del proyecto para la pertinencia de la educación primaria ha sido el dotarla de un espacio curricular para la lengua maya y atender los diferentes niveles de competencia del alumnado. A pesar de ser escuelas que se ubican en comunidades mayas, ninguna de las dos forma parte del subsistema indígena, como ya mencionamos; las clases de maya obedecen a un derecho lingüístico de los niños mayas. El taller de lengua maya ha permitido visibilizar los saberes lingüísticos de niños y niñas mayahablantes, que, coincidentemente, no suelen obtener los mejores resultados en las evaluaciones escolares desde la perspectiva de los docentes.

La construcción de relevancia de la educación se ha fomentado con la implementación del proyecto a través de la articulación de sus talleres con los objetivos de aprendizaje propuestos en los planes de estudio. La incursión en actividades artísticas, con la tierra y en su propia lengua ha demostrado a los docentes beneficios en cuanto a los aprendizajes de los niños y las niñas. Las ventajas de esta articulación han fortalecido el proyecto y afianzado un proceso que integre más sus actividades a las escolares. Así, podemos vislumbrar que la relevancia de la educación se conecta con la pertinencia y la equidad si los tres criterios se abordan desde una perspectiva cualitativa que apunte a una educación aceptable y adaptable, y no solo accesible y disponible.

Los logros en materia de equidad tienen un carácter también cualitativo que es necesario visibilizar. Si bien han sido posibles gracias a los recursos aportados por el proyecto, por ejemplo, para la compra de instrumentos musicales y el pago a los talleristas, se expresan en cambios en las expectativas de presente y futuro de todos los actores involucrados en el proceso educativo. La prospectiva de todos los niños y las niñas se ha transformado al ser parte de un proyecto que les ha permitido acceder a talleres que detonan aprendizajes in-esperados en un contexto vulnerado 
como el de estas comunidades. De ese modo, los aprendizajes que fomenta el proyecto no deben limitarse a los prejuicios que, convencionalmente, se han formulado en torno a los niños y las niñas indígenas cuyo derecho a la educación de calidad ha sido obstaculizado o negado.

\section{REFERENCIAS BIBLIOGRÁFICAS}

Blanco Bosco, E. (2019). ¿Cómo debe entenderse la equidad en educación en cada nivel y modalidad educativa? (educación indígena). En A. Buendía Espinosa y G. Âlvarez Mendiola (coords.). La investigación educativa ante el cambio de gobierno en México. Reflexiones y propuestas para el futuro (pp. 30-35). México: COMIE.

Cabrera García, F. (2019). Literacidades y pertinencia cultural en la asignatura de lengua indígena en primarias multigrado de Tequila, Sierra de Zongolica, México (tesis de maestría). Universidad Veracruzana, México. Recuperado de https://www.uv.mx/iie/files/2019/04/Tesis-Itzel-Cabrera.pdf

Cabrera Pacheco, A. J. (2014). Estrategias de sustentabilidad en el solar maya yucateco en Mérida, México. GeoGraphos, vol. 5, núm. 56, pp. 1-32. https:// doi.org/10.14198/GEOGRA2014.5.56

Denzin, N. K. (1970). The research act: A theoretical introduction to sociological methods. Nueva Jersey: Transaction Publishers.

Díaz-Barriga, F. (2003). Cognición situada y estrategias para el aprendizaje significativo. Revista Electrónica de Investigación Educativa, vol. 5, núm. 2, pp. 1-13. Recuperado de https://redie.uabc.mx/redie/article/view/85

Flick, U. (2004). Introducción a la investigación cualitativa. Madrid: Ediciones Morata.

Guba Egon, G. y Lincoln, Y. S. (2012). Controversias paradigmáticas, contradicciones y confluencias emergentes. En N. K. Denzin y Yvonna S. Lincoln (comps.). Paradigmas y perspectivas en disputa (pp. 38-78). Barcelona: Gedisa.

INEGI (2016). Panorama sociodemográfico de Yucatán 2015. México. Recuperado de http://internet.contenidos.inegi.org.mx/contenidos/Productos/prod serv/contenidos/espanol/bvinegi/productos/nueva_estruc/inter_censal/ panorama/702825082437.pdf

Jiménez Naranjo, Y. (2009). Cultura comunitaria y escuela intercultural. México: CGEIB, SEP.

Kvale, S. (1998). Ten standard objections to qualitative research interviews. Journal of Phenomenological Psychology, vol. 25, núm. 2, pp. 147-173.

Lomax, H. (2012). Contested voices? Methodological tensions in creative visual research with children. International Journal of Social Research Methodology, vol. 15, núm. 2, pp. 105-117.

Mejen t'aano'ob, Pequeñas Voces AC (s.f.). Proyecto “Arte, medio ambiente, conocimientos universales y locales claves para transformar la educación desde la comunidad" (documento interno).

Mendoza Zuany, R. G. (2019). ¿Cómo debe entenderse la equidad en educación en cada nivel y modalidad educativa? (educación indígena). En A. Buendía Espinosa y G. Álvarez Mendiola (coords.). La investigación educativa ante el cambio de gobierno en México. Reflexiones y propuestas para el futuro (pp. 4551). México: COMIE. 
Mendoza Zuany, R. G. (2020). Los aprendizajes in-esperados en casa para el mundo post-Covid19. Blog Mujeres Unidas por la Educación. Recuperado de https://www.muxed.mx/post/los-aprendizajes-in-esperados-en-casa-parael-mundo-post-covid19

Richardson, L. (1997). Fields of play: Constructing an academic life. New Brunswick: Rutgers University Press.

Richardson, L. y Adams St. Pierre, E. (2006). La escritura: un método de investigación. En N. K. Denzin y Y. S. Lincoln (comps.). El arte y la práctica de la interpretación, la evaluación y la presentación (pp. 128-163). Barcelona: Gedisa.

Schmelkes, S. (2018). Definiciones de calidad de la educación en el Instituto Nacional para la Evaluación de la Educación. Gaceta de la Política Nacional de Evaluación Educativa en México, núm. 10, pp. 18-22. Recuperado de https:// www.inee.edu.mx/etiqueta/gaceta-no-10/

Schmelkes, S. (2003). Educación para un México intercultural. Sinéctica, Revista Electrónica de Educación, núm. 40, pp. 1-12. Recuperado de https://sinectica. iteso.mx/index.php/SINECTICA/article/view/48

Sedesol (2013). Catálogo de localidades. Resumen municipal. Municipio de Cantamayec. México. Recuperado de http://www.microrregiones.gob.mx/catloc/LocdeMun.aspx?tipo=clave \&campo=loc\&ent $=31 \&$ mun $=010$

Tomasevsky, K. (2004). Indicadores del derecho a la educación. Revista IIDH, vol. 40, pp. 341-388. Recuperado de http://www.corteidh.or.cr/tablas/ R0806411.pdf

Unesco (2007). Educación de calidad para todos: un asunto de derechos humanos. Santiago de Chile: OREALC/Unesco. 